\title{
Uniform stability of the Dirichlet spectrum for rough outer perturbations
}

\author{
Bruno Colbois, Alexandre Girouard, and Mette Iversen
}

\begin{abstract}
The goal of this paper is to study the Dirichlet eigenvalues of bounded domains $\Omega \subset \Omega^{\prime}$. With a local spectral stability requirement on $\Omega$, we show that the difference of the Dirichlet eigenvalues of $\Omega^{\prime}$ and $\Omega$ is explicitly controlled from above in terms of the first eigenvalue of $\Omega^{\prime} \backslash \bar{\Omega}$ and of geometric constants depending on the inner domain $\Omega$. In particular, $\Omega^{\prime}$ can be an arbitrary bounded domain.
\end{abstract}

Mathematics Subject Classification (2010). Primary 35P99; Secondary 58J50.

Keywords. Spectral stability, Dirichlet spectrum.

\section{Introduction and results}

Let $M$ be a complete smooth Riemannian manifold. The Dirichlet eigenvalues of a bounded domain ${ }^{1} \Omega \subset M$ are denoted

$$
\lambda_{1}(\Omega)<\lambda_{2}(\Omega) \leq \lambda_{3}(\Omega) \leq \cdots \nearrow \infty .
$$

In the Euclidean case, there is a vast literature on spectral stability of the Dirichlet spectrum under perturbation of the domain. The aim is to show that if $\Omega^{\prime}$ is another domain which is, in some sense, geometrically close to $\Omega$, then its Dirichlet eigenvalues are close to those of $\Omega$. See for instance the papers [6] and [10] and the references therein, where spectral stability is studied in particular from the point of view of $\gamma$-convergence. Explicit control of the spectrum has been studied for example in [4] and [13].

In this paper, we are interested in obtaining explicit estimates in the situation where the two domains $\Omega$ and $\Omega^{\prime}$ might not be geometrically close. For domains $\Omega \subset \Omega^{\prime}$, the difference of eigenvalues $\left|\lambda_{k}(\Omega)-\lambda_{k}\left(\Omega^{\prime}\right)\right|$ will be controlled in terms of the fundamental tone $\lambda_{1}\left(\Omega^{\prime} \backslash \bar{\Omega}\right)$. In particular, the domains $\Omega^{\prime}$ and $\Omega$ can have very different shapes, and the volume of $\Omega^{\prime}$ can be large compared to that of $\Omega$. Some natural conditions on the inner domain $\Omega$ need to be assumed, but $\Omega^{\prime}$ can be

\footnotetext{
${ }^{1} \mathrm{~A}$ domain is a connected open set.
} 
any bounded open set. We will do this by combining local estimates based on the work of E.B. Davies [8], with global estimates based on the work of the first author and J. Bertrand [3]. Note that in this paper constants will depend only on the stated parameters.

1.1. Statement of results. Our goal is to estimate $\left|\lambda_{k}(\Omega)-\lambda_{k}\left(\Omega^{\prime}\right)\right|$ in the situation where $\Omega \subset \Omega^{\prime}$ are bounded domains. Throughout we will use the notation

$$
\Omega^{\varepsilon}=\{x \in M: d(x, \Omega)<\varepsilon\}, \quad \Omega_{\varepsilon}=\left\{x \in \Omega: d\left(x, \Omega^{c}\right)>\varepsilon\right\},
$$

and

$$
\mu=\lambda_{1}\left(\Omega^{\prime} \backslash \bar{\Omega}\right), \quad \lambda=\lambda_{k}\left(\Omega^{\prime}\right),
$$

with the convention $\lambda_{1}(\emptyset)=\infty$. Observe that because $\Omega \subset \Omega^{\varepsilon} \cap \Omega^{\prime} \subset \Omega^{\prime}$, it follows from monotonicity of the Dirichlet eigenvalues that

$$
0 \leq \lambda_{k}(\Omega)-\lambda_{k}\left(\Omega^{\prime}\right)=\left(\lambda_{k}\left(\Omega^{\varepsilon} \cap \Omega^{\prime}\right)-\lambda_{k}\left(\Omega^{\prime}\right)\right)+\left(\lambda_{k}(\Omega)-\lambda_{k}\left(\Omega^{\varepsilon} \cap \Omega^{\prime}\right)\right),
$$

where the two terms on the right hand side are non-negative. We estimate these two terms separately, and call them the global and the local term respectively.

Global estimates. In Section 2, we prove the following theorem allowing control of the first term in the right hand side of (1.1) when $\mu=\lambda_{1}\left(\Omega^{\prime} \backslash \bar{\Omega}\right)$ is large.

Theorem 1.1. There are constants $a_{k}, b_{k} \geq 1$ defined by the recurrence relations (2.1) and (2.2), with the following property. For each $\alpha \in\left(0, \frac{1}{4}\right)$, if

$$
32\left(\frac{\lambda}{\mu}\right)^{\frac{1}{2}-2 \alpha} a_{k} \leq 1
$$

then

$$
\left|\lambda_{k}\left(\Omega^{\varepsilon} \cap \Omega^{\prime}\right)-\lambda\right| \leq c_{k, \alpha}\left(\frac{\lambda}{\mu}\right)^{\frac{1}{2}-2 \alpha} \lambda
$$

for the choice

$$
\varepsilon=\left(\frac{\lambda}{\mu}\right)^{\alpha} \frac{1}{\sqrt{\lambda}}
$$

and with

$$
c_{k, \alpha}=2^{1 /(2 \alpha)} 32 b_{k} .
$$

The strategy of the proof consists in a geometrical approach inspired by a special case of the proof of Theorem 3.3 in [3]. 
Remark 1.1. Inequality (1.3) is invariant under homothetic scaling of the domains due to the specific choice of $\varepsilon$. This is important for the potential value to numerical analysis, and because the problem is naturally scaling invariant. However, this choice of $\varepsilon$ implies a need to bound $\lambda$ from below as we pass to the local estimate. This issue is discussed in Remark 1.3.

Local estimates. In Section 3, we describe classes of domains $\Omega \subset \mathbb{R}^{n}$ for which we have uniform control of the second term in the right hand side of (1.1) for each $k \in \mathbb{N}$. They consist of domains $\Omega$ with thickenings $\Omega^{\varepsilon}$ satisfying a weak Hardy inequality.

Definition 1.1. A domain $\Omega$ satisfies a weak Hardy inequality with constants $a, b$ if for each $u \in C_{0}^{\infty}(\Omega)$,

$$
\int_{\Omega} \frac{u^{2}}{\delta^{2}} \leq a \int_{\Omega}\left(|\nabla u|^{2}+b u^{2}\right)
$$

where $\delta$ denotes the distance function to the boundary of $\Omega$.

The following illustrates our use of the Hardy inequality for a particular class of domains. (See Section 3.4 for the definition of the uniform external rolling ball condition.)

Lemma 1.1. Let $\varepsilon_{0}>0, r_{0}>0$. Let $\mathcal{A}=\mathcal{A}\left(\varepsilon_{0}, r_{0}, n\right)$ be the family of open, bounded sets $\Omega$ in $\mathbb{R}^{n}, n \geq 2$, with inradius bounded below by $r_{0}$ satisfying a uniform external rolling ball condition with parameter $\varepsilon_{0}$. Then there exist positive constants $\gamma=\gamma(n), C_{k}=C_{k}\left(n, \varepsilon_{0}, r_{0}\right)$ and $\varepsilon_{k}=\varepsilon_{k}\left(n, \varepsilon_{0}, r_{0}\right)$ such that for any $\Omega \in \mathcal{A}$ and any $\varepsilon \leq \min \left(\varepsilon_{0} / 2, \varepsilon_{k}\right)$,

$$
0<\lambda_{k}(\Omega)-\lambda_{k}\left(\Omega^{\varepsilon}\right) \leq C_{k} \varepsilon^{\gamma}
$$

Remark 1.2. The proof of Lemma 1.1 is based on the fact that the geometric hypotheses imply uniform control of the constants $a$ and $b$ appearing in the Hardy inequalities for the thickenings $\Omega^{\varepsilon}$. This control allows the application of a result of E.B. Davies from [8]. For convex domains, we obtain scale invariant local bounds via an alternative geometric proof (see Proposition 3.1).

Corollary 1.1. Under the hypotheses of Lemma 1.1, there exist constants $a_{k}, b_{k}$, $\gamma=\gamma(n), C_{k}=C_{k}\left(n, \varepsilon_{0}, r_{0}\right)$ and $\varepsilon_{k}=\varepsilon_{k}\left(n, \varepsilon_{0}, r_{0}\right)$ such that for each domain $\Omega \in \mathcal{A}$ the following holds. Let $\alpha \in\left(0, \frac{1}{4}\right)$, and suppose that (1.2) holds and that

$$
\left(\frac{\lambda}{\mu}\right)^{\alpha} \frac{1}{\sqrt{\lambda}} \leq \min \left(\varepsilon_{0}, \varepsilon_{k}\right)
$$


holds. Then

$$
\left|\lambda_{k}(\Omega)-\lambda\right| \leq c_{k, \alpha}\left(\frac{\lambda}{\mu}\right)^{\frac{1}{2}-2 \alpha} \lambda+\frac{C_{k}}{\lambda^{\frac{\nu}{2}}}\left(\frac{\lambda}{\mu}\right)^{\gamma \alpha},
$$

where as above $\lambda=\lambda_{k}\left(\Omega^{\prime}\right)$ and $c_{k, \alpha}=2^{1 /(2 \alpha)} 32 b_{k}$. Taking for example $\alpha=\frac{1}{2(2+\gamma)}$ gives

$$
\left|\lambda_{k}(\Omega)-\lambda\right| \leq\left(c_{k, \alpha} \lambda^{\frac{4+3 \gamma}{2(2+\gamma)}}+C_{k} \lambda^{-\frac{\gamma(1+\gamma)}{2(2+\gamma)}}\right) \mu^{-\frac{\gamma}{2(2+\nu)}} .
$$

Remark 1.3. Monotonicity implies that the eigenvalue $\lambda=\lambda_{k}\left(\Omega^{\prime}\right)$ is bounded above by $\lambda_{k}(\Omega)$, so that the numerators of the right hand side of (1.3) and the first term in the right hand side of (1.5) are bounded above in terms of $\Omega$ and $k$ only. A more delicate issue is bounding $\lambda$ from below, which is relevant for the second term in the right hand side of (1.5). To ensure $\lambda$ is sufficiently large to have a meaningful estimate, it is sufficient to bound $\lambda_{1}\left(\Omega^{\prime}\right)$ from below. Such a bound is obtained for example by the Faber-Krahn inequality if we choose to bound the volume of $\Omega^{\prime}$ from above.

In Section 3, similar results will be proved for families defined in terms of a cone condition and a capacity density condition. In Section 3.1, we give two simple examples to illustrate the necessity of two of the geometric conditions imposed in Section 3.4. Our goal for this section is to give some simple criteria implying local stability.

Proximity of eigenspaces. In Section 4 , we control the proximity of the eigenspaces on $\Omega^{\prime}$ and $\Omega$ in terms of $\mu=\lambda_{1}\left(\Omega^{\prime} \backslash \bar{\Omega}\right)$. Stability of eigenfunctions for the Dirichlet problem is well known, so our contribution is to provide an explicit control in terms of $\mu$.

1.2. Discussion of results. Stability of the Dirichlet spectrum is closely related to the stability of the corresponding Dirichlet problem

$$
-\Delta u=f \text { in } \Omega, \quad u=0 \text { on } \partial \Omega .
$$

Indeed, it is well known that estimates for the associated resolvent operator $R_{\Omega}$ translate into corresponding bounds for the eigenvalues. This has been studied from the point of view of various interrelated notions of convergence of domains. See Chapter 2.3 in [11] for an enlightening discussion. In [6] it is proved, under rather weak assumptions on $\Omega$, that if a sequence $\Omega_{n}$ of domains containing $\Omega$ is such that

$$
\lim _{n \rightarrow \infty} \lambda_{1}\left(\Omega_{n} \backslash \bar{\Omega}\right)=\infty,
$$

then $\Omega_{n} \gamma$-converges to $\Omega$, which implies

$$
\lim _{n \rightarrow \infty} \lambda_{k}\left(\Omega_{n}\right)=\lambda_{k}(\Omega)
$$


In this situation, our results provide explicit control of the difference $\left|\lambda_{k}(\Omega)-\lambda_{k}\left(\Omega_{n}\right)\right|$ in terms of $\mu$, and so control of the rate of convergence.

As mentioned, explicit estimates of the difference $\left|\lambda_{k}(\Omega)-\lambda_{k}\left(\Omega^{\prime}\right)\right|$ in terms of for example the measure of the symmetric difference $\Omega^{\prime} \Delta \Omega$ in [4] have also been given previously. Our estimate in terms of $\mu=\lambda_{1}\left(\Omega^{\prime} \backslash \bar{\Omega}\right)$ allows control in addition when the measure of $\Omega^{\prime} \backslash \Omega$ is large. The results of [4] are valid for classes of Lipschitz domains described in Section 2.3 in [4]. Our results complement this by providing a selection of geometric conditions under which we have control of the spectrum.

Remark 1.4. In her $\mathrm{PhD}$ thesis [12], the third author has developed an alternative version of Theorem 1.1 and Corollary 1.1. The emphasis of this work is scaling invariance.

Acknowledgements. We would like to thank Michiel van den Berg, Dorin Bucur, Antoine Lemenant, Yuri Netrusov, and Iosif Polterovich for valuable discussions.

\section{Global estimates}

The goal of this section is to prove Theorem 1.1. Let $\Omega \subset \Omega^{\prime}$ be bounded domains in the complete smooth Riemannian manifold $M$. Let $\left(f_{i}\right)_{i \in \mathbb{N}}$ be an orthonormal basis of $L^{2}\left(\Omega^{\prime}\right)$ corresponding to the Dirichlet eigenvalues $\lambda_{i}\left(\Omega^{\prime}\right)$, which are extended by zero outside of $\Omega^{\prime}$. Fix $\varepsilon>0$ and let $\eta: M \rightarrow \mathbb{R}$ be a cutoff function such that

$$
\begin{gathered}
0 \leq \eta \leq 1, \quad|\nabla \eta| \leq \frac{1}{\varepsilon}, \\
\eta \equiv\left\{\begin{array}{l}
1 \text { in } \Omega^{\varepsilon}, \\
0 \text { in } M \backslash \Omega^{2 \varepsilon} .
\end{array}\right.
\end{gathered}
$$

For each $k \in \mathbb{N}$, the function

$$
\psi_{k} \stackrel{\text { def }}{=} \eta f_{k} \in H_{0}^{1}\left(\Omega^{2 \varepsilon} \cap \Omega^{\prime}\right)
$$

will be used as test function in the variational characterization of $\lambda_{k}\left(\Omega^{2 \varepsilon}\right)$ thanks to a result of the first author and J. Bertrand Lemma 3.13 in [3]. This result is stated here in a new scaling invariant version.

Lemma 2.1. Let $k \in \mathbb{N}$ be a fixed integer. Let $a_{1}=1$ and for $j \in\{2, \ldots, k\}$, recursively define

$$
a_{j}=1+\left(\sum_{i=1}^{j-1} a_{i}^{2}\right)^{2} .
$$


Let $\rho_{j}$ be a positive number such that $4 \rho_{j} a_{j} \leq 1$. Let $b_{1}=4$ and for $j \in\{2, \ldots, k\}$, recursively define

$$
b_{j}=\left(1+8 a_{j}\right)\left(\left(1+b_{j-1}\right)\left(1+8 a_{j}\right)+1\right) .
$$

For each $\lambda>0$, the following holds: Let $q$ be a quadratic form on a Euclidean space $E$ of dimension $k$. Let $\psi_{1}, \ldots, \psi_{k} \in E$ be such that for each $i, j \leq k$,

$$
\left|\left\langle\psi_{i}, \psi_{j}\right\rangle-\delta_{i j}\right| \leq \rho_{k} \quad \text { and } \quad q\left(\psi_{i}\right) \leq \lambda\left(1+\rho_{k}\right) .
$$

Then there exists an orthonormal basis $\left(F_{i}\right)_{1 \leq i \leq k}$ of $E$ such that for each $i \in$ $\{1,2, \ldots, k\}$

$$
q\left(F_{i}\right) \leq \lambda\left(1+\rho_{k} b_{k}\right) .
$$

The proof of Lemma 2.1 differs only slightly from the original proof, and the modifications will be presented in Section 5. In our situation, the quadratic form is the Dirichlet energy defined on the space $E=\operatorname{span}\left(\psi_{1}, \ldots, \psi_{k}\right)$ in $L^{2}\left(\Omega^{2 \varepsilon} \cap \Omega^{\prime}\right) \subset$ $L^{2}(M)$.

Lemma 2.2. For each $j \in\{1,2, \ldots, k\}$, the Dirichlet energy of the test function $\psi_{j}=\eta f_{j}$ satisfies

$$
\left\|\nabla \psi_{j}\right\|^{2} \leq \lambda+\Lambda+2 \Lambda^{1 / 2} \lambda^{1 / 2},
$$

where $\Lambda=2\left(\frac{1+\varepsilon^{2} \lambda}{\varepsilon^{4} \mu}\right)$.

Proof of Lemma 2.2. Writing $f=f_{j}$ and $\psi=\eta f$ to simplify notations, direct computation using the definition of $\psi$ and of the cutoff function $\eta$ leads to

$$
\begin{aligned}
\int_{\Omega^{2 \varepsilon}}|\nabla \psi|^{2} & =\int_{\Omega^{2 \varepsilon} \backslash \Omega^{\varepsilon}}|\nabla \eta|^{2} f^{2}+2 \int_{\Omega^{2 \varepsilon} \backslash \Omega^{\varepsilon}} \eta f \nabla \eta \cdot \nabla f+\int_{\Omega^{2 \varepsilon}} \eta^{2}|\nabla f|^{2} \\
& \leq \frac{1}{\varepsilon^{2}} \underbrace{\int_{\Omega^{2 \varepsilon} \backslash \Omega^{\varepsilon}}}_{A} f^{2}+\frac{2}{\varepsilon} \int_{\Omega^{2 \varepsilon} \backslash \Omega^{\varepsilon}}|f||\nabla f|+\int_{\Omega^{2 \varepsilon}}|\nabla f|^{2} \\
& \leq \frac{1}{\varepsilon^{2}} A+\frac{2}{\varepsilon} A^{1 / 2}\left(\int_{\Omega^{2 \varepsilon} \backslash \Omega^{\varepsilon}}|\nabla f|^{2}\right)^{1 / 2}+\int_{\Omega^{2 \varepsilon}}|\nabla f|^{2} \\
& \leq \frac{1}{\varepsilon^{2}} A+\frac{2}{\varepsilon} A^{1 / 2} \lambda^{1 / 2}+\lambda .
\end{aligned}
$$

In order to give an upper bound for

$$
A=\int_{\Omega^{2 \varepsilon} \backslash \Omega^{\varepsilon}} f^{2},
$$


an auxiliary cutoff function

$$
\chi: M \longrightarrow \mathbb{R}
$$

is introduced, satisfying

$$
\begin{gathered}
0 \leq \chi \leq 1, \quad|\nabla \chi| \leq \frac{1}{\varepsilon}, \\
\chi \equiv\left\{\begin{array}{l}
1 \text { in } M \backslash \Omega^{\varepsilon}, \\
0 \text { in } \Omega .
\end{array}\right.
\end{gathered}
$$

It follows that

$$
A=\int_{\Omega^{2 \varepsilon} \backslash \Omega^{\varepsilon}} \chi^{2} f^{2} \leq \int_{M \backslash \Omega} \chi^{2} f^{2}=\|\chi f\|^{2} .
$$

The function $\chi f$ is then used in the variational characterization of $\mu$ :

$$
\begin{aligned}
\mu\|\chi f\|^{2} & \leq \int_{\Omega^{\prime} \backslash \bar{\Omega}}|\nabla(\chi f)|^{2} \leq 2 \int_{\Omega^{\prime} \backslash \bar{\Omega}}\left(|\nabla \chi|^{2} f^{2}+\chi^{2}|\nabla f|^{2}\right) \\
& \leq \frac{2}{\varepsilon^{2}} \int_{\Omega^{\prime} \backslash \bar{\Omega}} f^{2}+2 \int_{\Omega^{\prime} \backslash \bar{\Omega}}|\nabla f|^{2} \\
& \leq 2\left(\frac{1}{\varepsilon^{2}}+\lambda\right) .
\end{aligned}
$$

It follows that

$$
A \leq\|\chi f\|^{2} \leq 2\left(\frac{1+\varepsilon^{2} \lambda}{\varepsilon^{2} \mu}\right),
$$

which is substituted back into inequality (2.5) to complete the proof.

The following lemma shows that the test functions $\psi_{i}$ form an almost orthonormal family in $L^{2}$.

Lemma 2.3. For each $i, j \leq k$,

$$
\left|\left\langle\psi_{i}, \psi_{j}\right\rangle-\delta_{i j}\right| \leq 8 \varepsilon^{2} \Lambda,
$$

where $\Lambda=2\left(\frac{1+\varepsilon^{2} \lambda}{\varepsilon^{4} \mu}\right)$.

Proof of Lemma 2.3. For the case $i=j$, the inequality (2.7) implies

$$
\left\|\psi_{i}\right\|^{2} \geq \int_{\Omega^{\varepsilon}} f_{i}^{2}=1-\int_{M \backslash \Omega^{\varepsilon}} \chi^{2} f_{i}^{2} \geq 1-2\left(\frac{1+\varepsilon^{2} \lambda}{\varepsilon^{2} \mu}\right),
$$


where the cutoff function $\chi$ has been defined in (2.6). Since $\left\|\psi_{i}\right\|^{2} \leq\left\|f_{i}\right\|^{2}=1$, this implies

$$
\left|\left\langle\psi_{i}, \psi_{i}\right\rangle-1\right| \leq 2\left(\frac{1+\varepsilon^{2} \lambda}{\varepsilon^{2} \mu}\right)=\varepsilon^{2} \Lambda .
$$

For $i \neq j$,

$$
\left|\left\langle\psi_{i}, \psi_{j}\right\rangle\right|=\left|\int_{\Omega^{2 \varepsilon}} \eta^{2} f_{i} f_{j}\right|=\left|\int_{\Omega^{2 \varepsilon}}\left(\eta^{2}-1\right) f_{i} f_{j}+\int_{\Omega^{2 \varepsilon}} f_{i} f_{j}\right| .
$$

As $f_{i}$ and $f_{j}$ are orthogonal on $\Omega^{\prime}$ and extended by zero outside of $\Omega^{\prime}$,

$$
\left|\int_{\Omega^{2 \varepsilon}} f_{i} f_{j}\right|=\left|\int_{\Omega^{\prime} \backslash \Omega^{2 \varepsilon}} f_{i} f_{j}\right| \leq 2 \int_{\Omega^{\prime} \backslash \Omega^{2 \varepsilon}}\left(f_{i}^{2}+f_{j}^{2}\right) \leq 2\left(\left\|\chi f_{i}\right\|^{2}+\left\|\chi f_{j}\right\|^{2}\right),
$$

which together with $1-\eta^{2} \leq \chi^{2}$ implies

$$
\left|\int_{\Omega^{2 \varepsilon}}\left(\eta^{2}-1\right) f_{i} f_{j}\right| \leq 2\left(\left\|\chi f_{i}\right\|^{2}+\left\|\chi f_{j}\right\|^{2}\right) .
$$

Combining this with inequality (2.7) and noting that $\max \left(\lambda_{i}, \lambda_{j}\right) \leq \lambda_{k}\left(\Omega^{\prime}\right)$, then gives

$$
\left|\int_{\Omega^{2 \varepsilon}} \psi_{i} \psi_{j}\right| \leq 16 \frac{1+\varepsilon^{2} \lambda}{\varepsilon^{2} \mu}
$$

Proof of Theorem 1.1. Given $\alpha \in\left(0, \frac{1}{4}\right)$, let

$$
\varepsilon=\left(\frac{\lambda}{\mu}\right)^{\alpha} \frac{1}{\sqrt{\lambda}} .
$$

Note that because $a_{k}>1$ by definition, it follows from condition (1.2) that $\varepsilon<1 / \sqrt{\lambda}$, which implies $\Lambda \leq \frac{4}{\varepsilon^{4} \mu}$. Thus

$$
\begin{aligned}
\Lambda+2 \sqrt{\Lambda} \sqrt{\lambda} & \leq \frac{4}{\varepsilon^{4} \mu}+\frac{4 \sqrt{\lambda}}{\varepsilon^{2} \sqrt{\mu}}=4\left(\frac{\lambda}{\mu}\right)^{1-4 \alpha} \lambda+4\left(\frac{\lambda}{\mu}\right)^{\frac{1}{2}-2 \alpha} \lambda \\
& \leq 8\left(\frac{\lambda}{\mu}\right)^{\frac{1}{2}-2 \alpha} \lambda .
\end{aligned}
$$

Moreover

$$
\varepsilon^{2} \Lambda \leq \frac{4}{\varepsilon^{2} \mu}=4\left(\frac{\lambda}{\mu}\right)^{1-2 \alpha} \leq 8\left(\frac{\lambda}{\mu}\right)^{\frac{1}{2}-2 \alpha} .
$$

By condition (1.2) of Theorem 1.1, the hypotheses of Lemma 2.1 then hold with

$$
\rho=8\left(\frac{\lambda}{\mu}\right)^{\frac{1}{2}-2 \alpha} .
$$


This gives

$$
\left|\lambda_{k}\left(\Omega^{2 \varepsilon} \cap \Omega^{\prime}\right)-\lambda\right| \leq 8 b_{k}\left(\frac{\lambda}{\mu}\right)^{\frac{1}{2}-2 \alpha} \lambda,
$$

and so

$$
\left|\lambda_{k}\left(\Omega^{\varepsilon} \cap \Omega^{\prime}\right)-\lambda\right| \leq c_{k, \alpha}\left(\frac{\lambda}{\mu}\right)^{\frac{1}{2}-2 \alpha} \lambda,
$$

for $c_{k, \alpha}=2^{1 /(2 \alpha)} 32 b_{k}$, as required.

\section{Uniform local stability}

A family $\mathcal{A}$ of domains $\Omega \subset M$ is uniformly locally stable if there exists $\varepsilon_{0}>0$ such that for each $\Omega \in \mathcal{A}$, a uniform upper bound for $\left|\lambda_{k}(\Omega)-\lambda_{k}\left(\Omega^{\varepsilon}\right)\right|$ holds, in terms of $\varepsilon \leq \varepsilon_{0}, k \in \mathbb{N}$, and some geometric quantities depending only on $\Omega$. The family $\mathcal{A}$ described in the introduction (see Lemma 1.1) is a prototypical example of uniform local stability. Other examples of such families will be given in Section 3.4.

3.1. Motivating examples. The goal of the present section is to give two simple examples to illustrate the necessity of the geometric conditions imposed in the construction of the families in Section 3.4. The first illustrates the need for a lower bound on the inradius, while the second for a condition of the form $\left(\Omega^{\varepsilon}\right)_{N \varepsilon} \subset \Omega$ for all $\varepsilon \leq \varepsilon_{0}$, as required in Lemma 1.1.

Example 1. The Dirichlet eigenvalues of the ball $\Omega=B(0, r) \subset \mathbb{R}^{n}$ are

$$
\lambda_{k}(\Omega)=\frac{c(n, k)}{r^{2}}, \quad k \in \mathbb{N},
$$

where $c(n, k)$ is the $k$-th eigenvalue of a ball of radius one in $\mathbb{R}^{n}$. It follows that

$$
\lambda_{k}(\Omega)-\lambda_{k}\left(\Omega^{\varepsilon}\right)=c(n, k) \varepsilon\left(\frac{2 r+\varepsilon}{r^{2}(\varepsilon+r)^{2}}\right) .
$$

In order to have a uniform upper bound on $\lambda_{k}(\Omega)-\lambda_{k}\left(\Omega^{\varepsilon}\right)$, it is necessary to consider balls $B(0, r)$ of radius $r$ bounded below, say by $r_{0}>0$. Consider the family

$$
\mathcal{A}=\mathcal{A}\left(n, r_{0}\right)=\left\{B(p, r): p \in \mathbb{R}^{n}, r \geq r_{0}\right\} .
$$

For any ball $\Omega \in \mathcal{A}$, and for any $\varepsilon \leq \varepsilon_{0} \stackrel{\text { def }}{=} r_{0}$, one easily sees that

$$
\lambda_{k}(\Omega)-\lambda_{k}\left(\Omega^{\varepsilon}\right) \leq \frac{3 c(n, k)}{r_{0}^{2}} \frac{\varepsilon}{r_{0}} .
$$

In the more general context of Section 3.4, this will translate into lower bounds on the inradius. 
Example 2. Consider the family $\mathcal{A}=\left\{\Omega_{t}: t \in(0,1)\right\}$ described in Figure 1. Some of the features of this family are:

(1) The inradius of $\Omega_{t}$ is uniformly bounded.

(2) The boundary $\partial \Omega_{t}$ is smooth and its curvature is uniformly bounded.

In spite of these two properties, this family is not uniformly locally stable. Indeed, for each $\varepsilon_{0}>0$, choosing $t \leq \varepsilon_{0}$ leads to discontinuous variations of $\lambda_{k}$ as $\varepsilon$ varies from 0 to $\varepsilon_{0}$. This follows from the fact that $\Omega_{t}^{\varepsilon}$ is completely different from $\Omega_{t}$ since at $\varepsilon=\varepsilon_{0} / 2$, the domain $\Omega_{t}^{\varepsilon}$ becomes doubly connected.

In Lemma 1.1, this situation was avoided by requiring $\left(\Omega^{\varepsilon}\right)_{N \varepsilon} \subset \Omega$ for all $\varepsilon \leq \varepsilon_{0}$, and we will need a condition of this type to be satisfied throughout.

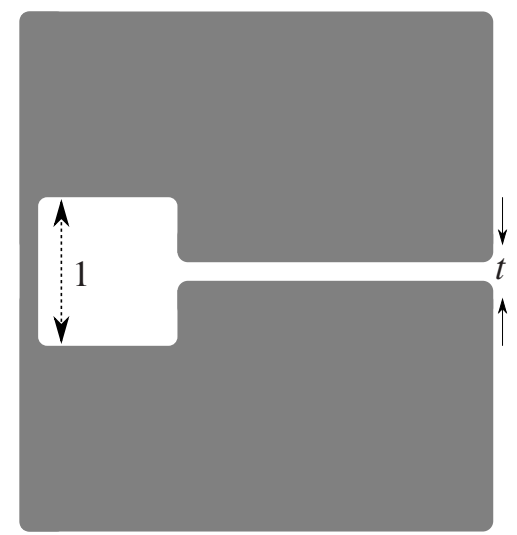

Figure 1. The domains $\Omega_{t}, 0<t<1$.

3.2. Convex domains. Local spectral stability is particularly simple for convex domains. Given $r_{0}>0$, consider the family

$$
\mathcal{A}=\mathcal{A}\left(n, r_{0}\right)=\left\{\Omega \subset \mathbb{R}^{n}: \Omega \text { is convex, } \operatorname{inradius}(\Omega) \geq r_{0}\right\} .
$$

Proposition 3.1. For any domain $\Omega \in \mathcal{A}$,

$$
\lambda_{k}(\Omega)-\lambda_{k}\left(\Omega^{\varepsilon}\right) \leq \frac{c(n, k)}{r_{0}^{2}}\left(\frac{2 \varepsilon}{r_{0}}+\frac{\varepsilon^{2}}{r_{0}^{2}}\right) .
$$

Lemma 3.1. Let $\Omega$ be a convex domain. Then $\left(\Omega^{\varepsilon}\right)_{\varepsilon}=\Omega$.

Proof of Lemma 3.1. A point $x \in \mathbb{R}^{n}$ lies in $\left(\Omega^{\varepsilon}\right)_{\varepsilon}$ if and only if $\overline{B(x, \varepsilon)} \subset \Omega^{\varepsilon}$. Suppose that $x \notin \Omega$. Then there exists a hyperplane separating $x$ from $\Omega$. This implies the existence of $y \in \overline{B(x, \varepsilon)}$ such that $d(y, \Omega) \geq \varepsilon$, which contradicts $\overline{B(x, \varepsilon)} \subset \Omega^{\varepsilon}$. 
Lemma 3.2. Let $r_{0}>0$. Let $\Omega \subset \mathbb{R}^{n}$ be a bounded convex Euclidean domain such that $B\left(x, r_{0}\right) \subset \Omega$. Then

$$
\Omega^{\varepsilon} \subset H(\Omega)
$$

where $H$ is an homothety of factor $1+\varepsilon / r_{0}$ with center $x$.

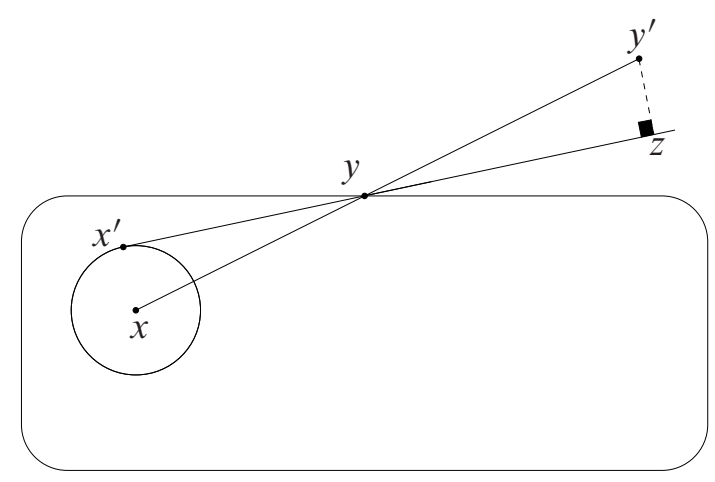

Figure 2. Proof of Lemma 3.2

Proof. Fix $y \in \partial \Omega$. There is a unique point $y^{\prime} \in \partial \Omega^{\varepsilon}$ such that $y \in x y^{\prime}$, where $x y^{\prime}$ denotes the segment connecting $x$ and $y^{\prime}$. There also exists a point $x^{\prime} \in \partial B\left(x, r_{0}\right)$ such that the segment $x^{\prime} y$ is tangent to $\partial B\left(x, r_{0}\right)$. Let $z$ be the orthogonal projection of $y^{\prime}$ onto the line through $x^{\prime}$ and $y$. It follows from Thales' theorem that we can compare the lengths of the segments to get

$$
\frac{\overline{z y^{\prime}}}{\overline{y y^{\prime}}}=\frac{\overline{x x^{\prime}}}{\overline{x y}}=\frac{r_{0}}{\overline{x y}} .
$$

Together with Lemma 3.1 this implies

$$
\frac{\overline{y y^{\prime}}}{\overline{x y}}=\frac{\overline{z y^{\prime}}}{r_{0}}<\frac{\varepsilon}{r_{0}} .
$$

Noting that $\overline{y y^{\prime}}=\overline{x y^{\prime}}-\overline{x y}$, we then have

$$
\overline{\frac{x y^{\prime}}{\overline{x y}}}<1+\frac{\varepsilon}{r_{0}} \text {. }
$$

This gives $y^{\prime} \in H(\Omega)$, and so completes the proof as $y \in \partial \Omega$ was arbitrary. 
3.3. A result of E. B. Davies. In studying the behavior of the spectrum of the outer perturbation $\Omega^{\varepsilon}$ we rely on a result of Davies [8] related to the stability of the inner perturbation $\Omega_{\varepsilon}$. In the sequel, we will consider bounded domains $\Omega \subset M$, where $M$ is a complete Riemannian manifold with Ricci curvature bounded from below by -1 . We will also denote by $r_{0}$ the inradius of $\Omega$, i.e. the radius of the largest ball contained in $\Omega$.

Theorem 3.1. Let $\Omega \subset M$ satisfy a weak Hardy inequality with constants $a, b$ and inradius bounded below by $r_{0}>0$. Then for each $k \in \mathbb{N}$, and each $0<\alpha \leq 1 / \sqrt{a}$, there exists constants $\varepsilon_{k}$ and $C_{k}$ depending only on $\alpha, a, b, r_{0}, k$, such that

$$
0 \leq \lambda_{k}\left(\Omega_{\varepsilon}\right)-\lambda_{k}(\Omega) \leq C_{k} \varepsilon^{2 \alpha},
$$

for any $0<\varepsilon<\varepsilon_{k}$.

The theorem is proved in [8] for more general operators, with $\Omega \subset \mathbb{R}^{n}$. In Theorem 13 in [9] Davies extends the result to include the sharp exponent. For insight we outline Davies' proof for the situation we are considering. The proof will make use of the following result proved in Theorem 14 in [8]. The fact that $c$ depends only on $a, \alpha$ can be deduced from a careful reading of the proofs in [8] and [9].

Theorem 3.2. Let $\Omega$ satisfy a weak Hardy inequality with constants $a, b$ and let $0<\alpha<1 / \sqrt{a}$. Then there exists a constant $c$ depending only on $a, \alpha$, such that for each $u \in \operatorname{Dom}(\Delta)$,

$$
\int_{\Omega} \frac{u^{2}}{\delta^{2+2 \alpha}} \leq c\|(-\Delta+b) u\|_{2}\left\|(-\Delta+b)^{1 / 2} u\right\|_{2},
$$

and

$$
\int_{\Omega} \frac{|\nabla u|^{2}}{\delta^{2 \alpha}} \leq c\|(-\Delta+b) u\|_{2}\left\|(-\Delta+b)^{1 / 2} u\right\|_{2},
$$

where $\delta$ again denotes the distance function to the boundary of $\Omega$.

Proof of Theorem 3.1. For each $k \in \mathbb{N}$, the span of the first $k$ eigenfunctions $\varphi_{i}, i=$ $1,2, \ldots, k$ of $\Omega$ is denoted $\Lambda_{k}$, and we write $\lambda_{k}=\lambda_{k}(\Omega)$ for the eigenvalues of $\Omega$. Given $u \in \Lambda_{k}$ with $\|u\|_{2}=1$, let $0<\varepsilon<r_{0} / 2$, and write $S=\Omega \backslash \Omega_{2 \varepsilon}$. Define the cut-off function $\chi: \Omega \rightarrow \mathbb{R}$ by

$$
\chi(x)= \begin{cases}0 & \text { if } x \in \Omega_{\varepsilon}^{c}, \\ \varepsilon^{-1} \operatorname{dist}\left(x, \partial \Omega_{\varepsilon}\right) & \text { if } x \in \Omega_{\varepsilon} \backslash \Omega_{2 \varepsilon}, \\ 1 & \text { if } x \in \Omega_{2 \varepsilon}\end{cases}
$$


Using that $\delta \leq 2 \varepsilon$ and $|\nabla \chi| \leq 1 / \varepsilon$ on $S$ leads to

$$
\begin{aligned}
\left.\left|\int_{\Omega}\right| \nabla(\chi u)\right|^{2}-\int_{\Omega}|\nabla u|^{2} \mid & \leq\left.\left|2\left(\int_{S}|\chi \nabla u|^{2}+\int_{S}|u \nabla \chi|^{2}\right)-\int_{\Omega}\right| \nabla u\right|^{2} \mid \\
& \leq 2 \int_{S}|u \nabla \chi|^{2}+\int_{S}|\nabla u|^{2} \\
& \leq \frac{2}{\varepsilon^{2}}(2 \varepsilon)^{2+2 \alpha} \int_{S} \frac{|u|^{2}}{\delta^{2+2 \alpha}}+(2 \varepsilon)^{2 \alpha} \int_{S} \frac{|\nabla u|^{2}}{\delta^{2 \alpha}} .
\end{aligned}
$$

Theorem 3.2 then gives

$$
\begin{aligned}
\left.\left|\int_{\Omega}\right| \nabla(\chi u)\right|^{2}-\int_{\Omega}|\nabla u|^{2} \mid & \leq \varepsilon^{2 \alpha}\left(2^{3+2 \alpha}+2^{2 \alpha}\right) c\|(-\Delta+b) u\|_{2}\left\|(-\Delta+b)^{1 / 2} u\right\|_{2} \\
& \leq \varepsilon^{2 \alpha} 2^{2 \alpha} 9 c\left(\lambda_{k}+b\right)^{3 / 2}\|u\|_{2}^{2}=\varepsilon^{2 \alpha} 2^{2 \alpha} 9 c\left(\lambda_{k}+b\right)^{3 / 2} .
\end{aligned}
$$

This holds for any $0<\alpha<1 / \sqrt{a}$ with $c=c(a, \alpha)$. For the last inequality we have used that $u \in \Lambda_{k}$ is of the form $u=\sum_{i=1}^{k} \alpha_{i} \varphi_{i}$ giving $-\Delta u=\sum_{i=1}^{k} \lambda_{i} \alpha_{i} \varphi_{i}$ for some $\alpha_{i} \in \mathbb{R}$, and thereby

$$
\begin{gathered}
\|(-\Delta+b) u\|_{2} \leq\left(\lambda_{k}+b\right)\|u\|_{2}, \\
\left\|(-\Delta+b)^{1 / 2} u\right\|_{2} \leq\left(\lambda_{k}+b\right)^{1 / 2}\|u\|_{2} .
\end{gathered}
$$

Next we estimate

$$
\begin{aligned}
\int_{\Omega} u^{2}-\int_{\Omega}(\chi u)^{2} & \leq \int_{S} u^{2} \leq \varepsilon^{2+2 \alpha} \int_{S} \frac{|u|^{2}}{\delta^{2+2 \alpha}} \\
& \leq \varepsilon^{2+2 \alpha} c\|(-\Delta+b) u\|_{2}\left\|(-\Delta+b)^{1 / 2} u\right\|_{2} \quad \text { (by Theorem 3.2) } \\
& \leq \varepsilon^{2+2 \alpha} c\left(\lambda_{k}+b\right)^{3 / 2} .
\end{aligned}
$$

Combining these two estimates with the min-max principle finally gives

$$
\begin{aligned}
\lambda_{k}\left(\Omega_{\varepsilon}\right) & \leq \sup \left\{\frac{\|\nabla v\|_{2}^{2}}{\|v\|_{2}^{2}}: v \in \operatorname{span}\left\{\chi \varphi_{1}, \ldots, \chi \varphi_{k}\right\}\right\} \\
& =\sup \left\{\frac{\|\nabla(\chi u)\|_{2}^{2}}{\|\chi u\|_{2}^{2}}: u \in \Lambda_{k}\right\} \\
& \leq \frac{\lambda_{k}+\varepsilon^{2 \alpha} 2^{2 \alpha} 9 c\left(\lambda_{k}+b\right)^{3 / 2}}{1-\varepsilon^{2+2 \alpha} c\left(\lambda_{k}+b\right)^{3 / 2}}
\end{aligned}
$$

If we take

$$
\varepsilon \leq\left(\frac{1}{2 c\left(\lambda_{k}+b\right)^{3 / 2}}\right)^{1 /(2+2 \alpha)} \stackrel{\text { def }}{=} \varepsilon_{k},
$$


we have

$$
\begin{aligned}
\lambda_{k}\left(\Omega_{\varepsilon}\right)-\lambda_{k} & \leq \frac{\varepsilon^{2 \alpha} 2^{2 \alpha} 9 c\left(\lambda_{k}+b\right)^{3 / 2}}{1-\varepsilon^{2+2 \alpha} c\left(\lambda_{k}+b\right)^{3 / 2}} \\
& \leq \varepsilon^{2 \alpha} 2^{2 \alpha+1} 9 c\left(\lambda_{k}+b\right)^{3 / 2}
\end{aligned}
$$

Using again that the inradius is bounded below by $r_{0}$, it follows from the proof of Corollary 2.3 in [5] that there exists a constant $\tilde{c}$ depending only on $n$ and $k$ such that

$$
\lambda_{k} \leq \frac{(n-1)^{2}}{4}+\frac{\tilde{c}}{r_{0}^{2}}
$$

Putting everything together, we have

$$
\lambda_{k}\left(\Omega_{\varepsilon}\right)-\lambda_{k} \leq C_{k}\left(\alpha, a, b, r_{0}\right) \varepsilon^{2 \alpha},
$$

for all $\varepsilon \leq \varepsilon_{k}=\varepsilon_{k}\left(\alpha, a, b, r_{0}\right)$. For the extension to the sharp exponent $\frac{2}{\sqrt{a}}$ please see Theorem 13 in [9].

3.4. Examples of locally stable families. In this section we construct families $\mathcal{A}$ of domains such that for each $k$ and each $\varepsilon>0$ small enough, the difference $\mid \lambda_{k}\left(\Omega^{\varepsilon}\right)-$ $\lambda_{k}(\Omega) \mid$ is controlled in terms of $\varepsilon, k$ and some geometric hypotheses on $\Omega$. The bounds are uniform in $\Omega \in \mathcal{A}$.

The goal is to describe families of domains for which we can use Theorem 3.1 to get such uniform estimates. Because Theorem 3.1 relates $\lambda_{k}(\Omega)$ to $\lambda_{k}\left(\Omega_{\varepsilon}\right)$ rather than to $\lambda_{k}\left(\Omega^{\varepsilon}\right)$, we will need the following conditions to hold for some $\varepsilon_{0}>0$ in addition to the lower bound $r_{0}$ on the inradius.

- There exists $N>0$ such that $\left(\Omega^{\varepsilon}\right)_{N \varepsilon}$ is contained in $\Omega$ for each $\varepsilon \leq \varepsilon_{0}$.

- The sets $\Omega^{\varepsilon}$ satisfy the weak Hardy inequality with constants $a, b$ independent of $\varepsilon \leq \varepsilon_{0}$.

This allows control of $\left|\lambda_{k}\left(\Omega^{\varepsilon}\right)-\lambda_{k}\left(\left(\Omega^{\varepsilon}\right)_{N \varepsilon}\right)\right|$, and so of $\left|\lambda_{k}\left(\Omega^{\varepsilon}\right)-\lambda_{k}(\Omega)\right|$ because $\left(\Omega^{\varepsilon}\right)_{N \varepsilon} \subset \Omega$. In the remainder of this section, we give an exposition of the situation in Euclidean space $\mathbb{R}^{n}$.

The following three definitions will be used.

Definition 3.1. An open set $\Omega \subset \mathbb{R}^{n}$ satisfies a uniform external cone condition [7], p. 129, with parameters $\alpha>0, \beta>0$ if for any $x \in \Omega^{c}$ we can find a cone $C$ of height $\beta$ and angle $\alpha$ with $x \in C$ and $C \subset \Omega^{c}$.

Definition 3.2. An open set $\Omega \subset \mathbb{R}^{n}$ satisfies a uniform external ball condition [2], p. 2, with parameters $\alpha>0, \beta>0$ if for any $z \in \partial \Omega$ and $0<r \leq \beta$ there exists $x \in \Omega^{c}$ with $d(z, x) \leq r$ such that $B(x, \alpha r) \subset \Omega^{c}$. It satisfies a uniform external rolling ball condition with parameter $\beta>0$ if this holds with $\alpha=1$. 
Definition 3.3. An open set $\Omega \subset \mathbb{R}^{n}, n \geq 3$ satisfies a uniform capacity density condition, see Lemma 3 in [1], with parameter $\alpha>0$ if for any $z \in \partial \Omega$ and any $r>0$

$$
\operatorname{cap}(B(z, r) \backslash \Omega) \geq \alpha r^{n-2} .
$$

Here cap is the capacity defined by

$$
\operatorname{cap}(\Gamma)=\inf \left\{\int_{\mathbb{R}^{n}}|\nabla v|^{2}: v \in C_{0}^{\infty}\left(\mathbb{R}^{n}\right), v \geq 1 \text { on } \Gamma\right\} .
$$

Remark 3.1. The uniform capacity density condition is weaker than the uniform external ball condition, which again is implied by the uniform external cone condition and finally by the stronger uniform Lipschitz condition. All of these conditions imply that the set satisfies a weak Hardy inequality.

\subsubsection{Uniform external rolling ball condition}

Proposition 3.2. Let $\varepsilon_{0}>0, r_{0}>0$. Let $\mathcal{A}=\mathcal{A}\left(\varepsilon_{0}, r_{0}, n\right)$ be a family of open, bounded sets $\Omega$ in Euclidean space $\mathbb{R}^{n}, n \geq 2$, with inradius bounded below by $r_{0}$. Then each $\Omega \in \mathcal{A}$ satisfies the uniform external rolling ball condition with parameter $\beta=\varepsilon_{0}$ if and only if $\left(\Omega^{\varepsilon}\right)_{\varepsilon} \subset \Omega$ for all $\varepsilon \leq \varepsilon_{0}$. In this case the sets $\Omega^{\varepsilon}, \varepsilon \leq \varepsilon_{0} / 2$, satisfy the uniform external rolling ball condition with parameter $\beta=\varepsilon_{0} / 2$.

Proof. Suppose $\left(\Omega^{\varepsilon}\right)_{\varepsilon} \subset \Omega$ for all $\varepsilon \leq \varepsilon_{0}$. Let $z \in \partial \Omega$ and $0<\varepsilon \leq \varepsilon_{0}$. Then as $\left(\Omega^{\varepsilon}\right)_{\varepsilon} \subset \Omega, z$ is not in $\left(\Omega^{\varepsilon}\right)_{\varepsilon}$, and so $d\left(z,\left(\Omega^{\varepsilon}\right)^{c}\right)=\varepsilon$. This gives $\overline{B(z, \varepsilon)} \cap\left(\Omega^{\varepsilon}\right)^{c} \neq$ $\emptyset$, and so we choose $x$ in this set. Then $x \in\left(\Omega^{\varepsilon}\right)^{c}$ implies $d(x, \Omega) \geq \varepsilon$, and so we have $B(x, \varepsilon) \subset \Omega^{c}$ with $d(z, x) \leq \varepsilon$ as required.

Now suppose $\Omega$ satisfies the uniform external rolling ball condition with parameter $\beta=\varepsilon_{0}$. Let $z \in \Omega^{c}$ and $0<\varepsilon \leq \varepsilon_{0}$. By the rolling ball condition, there exists $x$ such that $d(z, x) \leq \varepsilon_{0}$ and $B\left(x, \varepsilon_{0}\right) \subset \Omega^{c}$. As $\varepsilon \leq \varepsilon_{0}$, we have $x \in\left(\Omega^{\varepsilon}\right)^{c}$ and so $d\left(z,\left(\Omega^{\varepsilon}\right)^{c}\right) \leq \varepsilon$. Thus $z$ is not in $\left(\Omega^{\varepsilon}\right)_{\varepsilon}$, and we conclude that $\left(\Omega^{\varepsilon}\right)_{\varepsilon} \subset \Omega$.

Now note that if $\left(\Omega^{\varepsilon}\right)_{\varepsilon} \subset \Omega$ for all $\varepsilon \leq \varepsilon_{0}$, then the sets $\Omega^{\varepsilon}, \varepsilon \leq \varepsilon_{0} / 2$, satisfy a condition of the form $\left(\left(\Omega^{\varepsilon}\right)^{\delta}\right)_{\delta} \subset \Omega^{\varepsilon}$ for all $\delta \leq \varepsilon_{0} / 2$. Hence they also satisfy the uniform external rolling ball condition with parameter $\beta=\varepsilon_{0} / 2$.

Lemma 3.3. Let $\varepsilon_{0}>0, r_{0}>0$. Let $\mathcal{A}=\mathcal{A}\left(\varepsilon_{0}, r_{0}, n\right)$ be the family of open, bounded sets $\Omega$ in $\mathbb{R}^{n}, n \geq 2$, with inradius bounded below by $r_{0}$ satisfying a uniform external rolling ball condition with parameter $\varepsilon_{0}$. Then there exist constants $\gamma=\gamma(n)$, $\varepsilon_{k}=\varepsilon_{k}\left(n, \varepsilon_{0}, r_{0}\right)$ and $C_{k}=C_{k}\left(n, \varepsilon_{0}, r_{0}\right)$ such that for any $\Omega \in \mathcal{A}$ and any $\varepsilon \leq \min \left(\varepsilon_{0} / 2, \varepsilon_{k}\right)$,

$$
0<\lambda_{k}(\Omega)-\lambda_{k}\left(\Omega^{\varepsilon}\right) \leq C_{k} \varepsilon^{\gamma} .
$$


Proof. For $\varepsilon<\varepsilon_{0} / 2$, the sets $\Omega^{\varepsilon}$ satisfy the rolling ball condition with parameter $\varepsilon_{0} / 2$ by Proposition 3.2. By the proof of Theorem 1.5.4 in [7], the sets satisfy the weak Hardy inequalities

$$
\int_{\Omega} \frac{u^{2}}{\delta^{2}} \leq a\left(\int_{\Omega}|\nabla u|^{2}+b u^{2}\right), \quad u \in C_{0}^{\infty}(\Omega)
$$

and

$$
\int_{\Omega^{\varepsilon}} \frac{u^{2}}{\delta^{2}} \leq a\left(\int_{\Omega^{\varepsilon}}|\nabla u|^{2}+b u^{2}\right), \quad u \in C_{0}^{\infty}\left(\Omega^{\varepsilon}\right), \varepsilon \leq \varepsilon_{0} / 2,
$$

with constants $b=\left(2 / \varepsilon_{0}\right)^{2}$ and

$$
a=\frac{n}{32} \int_{0}^{\pi / 6} \sin ^{n-2}(t) d t / \int_{0}^{\pi / 2} \sin ^{n-2}(t) d t .
$$

Here $\delta$ denotes the distance function to the boundary. Then by Theorem 3.1, eq. (3.4) follows with $\gamma \leq \frac{2}{\sqrt{a}}$.

Combining Lemma 3.1 with Proposition 3.2 leads to the following corollary.

Corollary 3.1. Let $\varepsilon_{0}>0, r_{0}>0$. Let $\mathcal{A}=\mathcal{A}\left(\varepsilon_{0}, r_{0}, n\right)$ be the family of open, bounded sets $\Omega$ in $\mathbb{R}^{n}, n \geq 2$, with inradius bounded below by $r_{0}$ and such that for each $\Omega \in \mathcal{A},\left(\Omega^{\varepsilon}\right)_{\varepsilon} \subset \Omega$ for all $\varepsilon \leq \varepsilon_{0}$. Then there exist constants $\gamma=\gamma(n)$, $\varepsilon_{k}=\varepsilon_{k}\left(n, \varepsilon_{0}, r_{0}\right)$ and $C_{k}=C_{k}\left(n, \varepsilon_{0}, r_{0}\right)$ such that for any $\Omega \in \mathcal{A}$ and any $\varepsilon \leq \min \left(\varepsilon_{0} / 2, \varepsilon_{k}\right)$,

$$
0<\lambda_{k}(\Omega)-\lambda_{k}\left(\Omega^{\varepsilon}\right) \leq C_{k} \varepsilon^{\gamma}
$$

\subsubsection{Capacity density condition}

Lemma 3.4. Let $N, \varepsilon_{0}, r_{0}>0$. Let $\mathcal{A}=\mathcal{A}\left(N, \varepsilon_{0}, r_{0}, \alpha, n\right)$ be the family of open, bounded sets $\Omega$ in $\mathbb{R}^{n}, n \geq 3$, such that $\left(\Omega^{\varepsilon}\right)_{N \varepsilon} \subset \Omega$ for all $\varepsilon \leq \varepsilon_{0}$. Suppose also $\Omega^{\varepsilon}$ satisfies a uniform capacity density condition with parameter $\alpha>0$, uniformly in $\varepsilon \leq$ $\varepsilon_{0}$. Then there exist constants $\gamma=\gamma(\alpha), \varepsilon_{k}=\varepsilon_{k}\left(n, r_{0}, \alpha\right)$ and $C_{k}=C_{k}\left(n, r_{0}, \alpha\right)$ such that for any $\Omega \in \mathcal{A}$ and any $\varepsilon \leq \min \left(\varepsilon_{0}, \varepsilon_{k}\right)$,

$$
0<\lambda_{k}(\Omega)-\lambda_{k}\left(\Omega^{\varepsilon}\right) \leq C_{k}(N \varepsilon)^{\gamma} .
$$

This follows by a combination of Theorem 4.2 in [8] with Proposition 1 and Lemma 3 in [1], which gives a Hardy inequality for sets satisfying a uniform capacity density condition. 


\subsubsection{Uniform external cone condition}

Lemma 3.5. Let $\varepsilon_{0}>0, r_{0}>0$. Let $\mathcal{A}=\mathcal{A}\left(\varepsilon_{0}, r_{0}, \alpha, \beta, n\right)$ be the family of open, bounded sets in $\mathbb{R}^{n}, n \geq 2$, with inradius bounded below by $r_{0}$ and such that for each $\Omega \in \mathcal{A}$, the sets $\Omega, \Omega^{\varepsilon}, \varepsilon \leq \varepsilon_{0}$ satisfy a uniform external cone condition with parameters $\alpha>0, \beta>0$. Then there exist constants $\gamma=\gamma(\alpha, \beta), \varepsilon_{k}=$ $\varepsilon_{k}\left(n, \alpha, \beta, r_{0}\right)$ and $C_{k}=C_{k}\left(n, \alpha, \beta, r_{0}\right)$ such that for any $\varepsilon \leq \min \left(\frac{\beta \tan (\alpha / 2)}{\tan (\alpha / 2)+1}, \varepsilon_{k}\right)$, and any $\Omega \in \mathcal{A}$,

$$
0<\lambda_{k}(\Omega)-\lambda_{k}\left(\Omega^{\varepsilon}\right) \leq C_{k} \varepsilon^{\gamma}
$$

Proof. As mentioned Lemma 3 in [1] gives a Hardy inequality for sets satisfying a uniform capacity density condition and hence also for sets satisfying the stronger uniform external cone condition. This ensures that a weak Hardy inequality exists uniformly for the sets $\Omega, \Omega^{\varepsilon}, \varepsilon \leq \varepsilon_{0}$. We now show $\left(\Omega^{\varepsilon}\right)_{N \varepsilon} \subset \Omega$ for some $N=$ $N(\alpha)$ uniformly in $\varepsilon \leq \frac{\beta \tan (\alpha / 2)}{\tan (\alpha / 2)+1} \stackrel{\text { def }}{=} \varepsilon^{*}$ by showing that $x \in \Omega^{c}$ implies $x \in$ $\left(\left(\Omega^{\varepsilon^{*}}\right)_{N \varepsilon^{*}}\right)^{c}$. Then (3.4) follows by Theorem 3.1.

Take $x \in \Omega^{c}$ and let $C \subset \Omega^{c}$ be a cone of height $\beta$ and angle $\alpha$ with $x \in C$. Such a cone contains a ball of radius $\varepsilon^{*}$ centered at a point $y$ for which we then have $y \in$ $\left(\Omega^{\varepsilon^{*}}\right)^{c}$ and $d(x, y) \leq \frac{\varepsilon^{*}}{\tan (\alpha / 2)}$. Thus $x \in\left(\left(\Omega^{\varepsilon^{*}}\right)_{N \varepsilon^{*}}\right)^{c}$ for $N \geq(\tan (\alpha / 2))^{-1}$.

\subsubsection{The case of $\mathbb{R}^{2}$}

Lemma 3.6. Let $N, \varepsilon_{0}, r_{0}>0$. Let $\mathcal{A}=\mathcal{A}\left(N, \varepsilon_{0}, r_{0}\right)$ be the family of open, bounded sets $\Omega$ in $\mathbb{R}^{2}$ such that $\Omega^{\varepsilon}$ is simply connected with inradius bounded below by $r_{0}$, and satisfies $\left(\Omega^{\varepsilon}\right)_{N \varepsilon} \subset \Omega$ for all $\varepsilon \leq \varepsilon_{0}$. Then there exist constants $C_{k}=C_{k}\left(r_{0}\right)$ and $\varepsilon_{k}=\varepsilon_{k}\left(r_{0}\right)$ such that for any $\Omega \in \mathcal{A}$ and any $\varepsilon \leq \min \left(\varepsilon_{0}, \varepsilon_{k}\right)$,

$$
0<\lambda_{k}(\Omega)-\lambda_{k}\left(\Omega^{\varepsilon}\right) \leq C_{k} \varepsilon^{1 / 2} .
$$

The inequality follows by Theorem 3.1 using that simply connected planar sets satisfy a Hardy inequality with $a=16, b=0$; see Theorem 1.5 .10 in [7]. Note that the sharp exponent for Theorem 3.1 in $\mathbb{R}^{2}$ was first given in [14].

\section{Proximity of eigenspaces}

Some notation. Denote by $\lambda_{i}=\lambda_{i}(\Omega)$ the eigenvalues of $\Omega$, and by $\lambda_{i}^{\prime}=\lambda_{i}\left(\Omega^{\prime}\right)$ the eigenvalues of $\Omega^{\prime}$. Most of this paper has been devoted to estimation of the difference $\lambda_{i}-\lambda_{i}^{\prime}$. We write

$$
\delta_{i}=\max _{j=1}^{i}\left(\lambda_{j}-\lambda_{j}^{\prime}\right) .
$$


We consider an orthonormal basis $\left(f_{i}^{\prime}\right)_{i=1}^{\infty}$ of eigenfunctions of $\Omega^{\prime}$ corresponding to the eigenvalues $\lambda_{i}^{\prime}$. For $k \geq 1$, let $E_{k}$ be the successive distinct eigenspaces for $\Omega$. Let $n_{k}=\operatorname{dim}\left(E_{k}\right)$ be the corresponding multiplicities and set $N_{0}=0$ and $N_{k}=n_{1}+\cdots+n_{k}$. In particular, this means that for each $k \in \mathbb{N}$,

$$
\lambda_{N_{k-1}+1}=\cdots=\lambda_{N_{k-1}+n_{k}}=\lambda_{N_{k}} .
$$

We also introduce the corresponding vector space $E_{k}^{\prime}$ spanned by the eigenfunctions $f_{N_{k-1}+1}^{\prime}, \ldots, f_{N_{k}}^{\prime}$ on $\Omega^{\prime}$. The projection operator $P_{k}: L^{2}\left(\Omega^{\prime}\right) \rightarrow E_{k}^{\prime}$ is defined, for $f=\sum_{j=1}^{\infty} a_{j} f_{j}^{\prime}$, by

$$
P_{k}(f)=\sum_{j=N_{k-1}+1}^{N_{k}} a_{j} f_{j}^{\prime} .
$$

The gaps between successive distinct eigenvalues of $\Omega$ are $\lambda_{N_{k}+1}-\lambda_{N_{k}}$, and we write

$$
\Lambda_{k}=\min _{j=1}^{k}\left(\lambda_{N_{j}+1}-\lambda_{N_{j}}\right)
$$

In particular, if $i \leq k$, we have the relation $\frac{\delta_{i}}{\Lambda_{i}} \leq \frac{\delta_{k}}{\Lambda_{k}}$ which will be used in the proof, and $\frac{\delta_{k}}{\Lambda_{k}} \leq \frac{1}{2}$ implies $\frac{\delta_{i}}{\Lambda_{i}} \leq \frac{1}{2}$ for all $i \leq k$.

We can now express the proximity of eigenspaces.

Proposition 4.1. There exists a sequence of constants $\left(A_{k}\right)_{k=1}^{\infty}$ such that if $\delta_{N_{k}+1} \leq$ $\frac{\Lambda_{k}}{2 A_{k}}$, then the following holds.

A. For each $f \in E_{k},\|f\|=1$, we have

$$
\left\|\left(I-P_{k}\right)(f)\right\|^{2}=1-\left\|P_{k} f\right\|^{2} \leq \frac{A_{k} \delta_{N_{k-1}+1}}{\Lambda_{k}} .
$$

B. For each $f^{\prime} \in E_{k}^{\prime},\left\|f^{\prime}\right\|=1$, there exist $f \in E_{k},\|f\|=1$ with

$$
\left\|f-f^{\prime}\right\|^{2} \leq \frac{4 A_{k} \delta_{N_{k-1}+1}}{\Lambda_{k}}
$$

C. For each $f \in E_{k+l}, l>0,\|f\|=1$, we have

$$
\left\|\left(P_{1}+\cdots+P_{k}\right)(f)\right\|^{2} \leq \frac{4\left(\sum_{i=1}^{k} A_{i}\right) \delta_{N_{k-1}+1}}{\Lambda_{k}} .
$$

Moreover, the constants are defined by the induction $A_{k}=2+8 \frac{\lambda_{N_{k-1}+1} \sum_{i=1}^{k-1} A_{i}}{\Lambda_{k}}$, and so depend on $k$ and the spectrum of $\Omega$.

We first show the following technical proposition. 
Proposition 4.2. Under the condition $\delta_{N_{i}+1} \leq \frac{1}{2} \Lambda_{i}$, we have, for $f \in E_{i},\|f\|=1$,

$$
\begin{aligned}
\left\|f-P_{i}(f)\right\|^{2} & =1-\left\|P_{i}(f)\right\|^{2} \\
& \leq \frac{2}{\Lambda_{i}}\left(\delta_{N_{i-1}+1}+\lambda_{N_{i}+1}^{\prime}\left\|P_{1}(f)+\cdots+P_{i-1}(f)\right\|^{2}\right) .
\end{aligned}
$$

Proof. We consider $f \in E_{i}$ and, applying $q$, we get the relations

$$
\begin{aligned}
\lambda_{N_{i-1}+1}-q\left(P_{i}(f)\right) & =\sum_{j<i} q\left(P_{j}(f)\right) \\
& \geq \sum_{j<i} q\left(P_{j}(f)\right)+\lambda_{N_{i}+1}^{\prime}\left(1-\sum_{j \leq i}\left\|P_{j}(f)\right\|^{2}\right)
\end{aligned}
$$

and

$$
\begin{aligned}
& \lambda_{N_{i-1}+1}-q\left(P_{i}(f)\right) \\
& =\lambda_{N_{i-1}+1}-\lambda_{N_{i-1}+1}^{\prime}+\left(1-\left\|P_{i}(f)\right\|^{2}\right) \lambda_{N_{i-1}+1}^{\prime} \\
& \quad-\left(q\left(P_{i}(f)\right)-\lambda_{N_{i-1}+1}^{\prime}\left\|P_{i}(f)\right\|^{2}\right) .
\end{aligned}
$$

Putting this together, we get

$$
\begin{gathered}
\lambda_{N_{i-1}+1}-\lambda_{N_{i-1}+1}^{\prime}+\left(1-\left\|P_{i}(f)\right\|^{2}\right) \lambda_{N_{i-1}+1}^{\prime} \\
\geq \sum_{j<i} q\left(P_{j}(f)\right)+\lambda_{N_{i}+1}^{\prime}\left(1-\sum_{j \leq i}\left\|P_{j}(f)\right\|^{2}\right) \\
+q\left(P_{i}(f)\right)-\lambda_{N_{i-1}+1}^{\prime}\left\|P_{i}(f)\right\|^{2} .
\end{gathered}
$$

Noticing that $q\left(P_{i}(f)\right)-\lambda_{N_{i-1}+1}^{\prime}\left\|P_{i}(f)\right\|^{2} \geq 0$, we get

$$
\begin{gathered}
\lambda_{N_{i-1}+1}-\lambda_{N_{i-1}+1}^{\prime}+\lambda_{N_{i}+1}^{\prime} \sum_{j<i}\left\|P_{j}(f)\right\|^{2} \\
\geq\left(1-\left\|P_{i}(f)\right\|^{2}\right)\left(\lambda_{N_{i}+1}^{\prime}-\lambda_{N_{i-1}+1}^{\prime}\right),
\end{gathered}
$$

i.e.

$$
\left(1-\left\|P_{i}(f)\right\|^{2}\right) \leq \frac{\left(\lambda_{N_{i-1}+1}-\lambda_{N_{i-1}+1}^{\prime}\right)+\lambda_{N_{i}+1}^{\prime} \sum_{j<i}\left\|P_{j}(f)\right\|^{2}}{\lambda_{N_{i}+1}^{\prime}-\lambda_{N_{i-1}+1}^{\prime}} .
$$

Moreover,

$$
\begin{aligned}
\lambda_{N_{i}+1}^{\prime}-\lambda_{N_{i-1}+1}^{\prime}= & \left(\lambda_{N_{i}+1}^{\prime}-\lambda_{N_{i}+1}\right)+\left(\lambda_{N_{i}+1}-\lambda_{N_{i-1}+1}\right) \\
& +\left(\lambda_{N_{i-1}+1}-\lambda_{N_{i-1}+1}^{\prime}\right) \\
\geq & \Lambda_{i}-\left(\lambda_{N_{i}+1}-\lambda_{N_{i}+1}^{\prime}\right) .
\end{aligned}
$$


So under the condition $\left(\lambda_{N_{i}+1}-\lambda_{N_{i}+1}^{\prime}\right) \leq \frac{1}{2} \Lambda_{i}$, we get

$$
\left(1-\left\|P_{i}(f)\right\|^{2}\right) \leq 2 \frac{\left(\lambda_{N_{i-1}+1}-\lambda_{N_{i-1}+1}^{\prime}\right)+\lambda_{N_{i}+1}^{\prime} \sum_{j<i}\left\|P_{j}(f)\right\|^{2}}{\Lambda_{i}} .
$$

Before proving Proposition 4.1, we recall a fact of linear algebra which is needed for the proof.

Lemma 4.1. Let $(V,\langle.,\rangle$.$) be a prehilbert vector space, P$ a projector and $v$ a vector of norm 1 such that $P(v) \neq 0$. Then we have

$$
\left\|v-\frac{P(v)}{\|P(v)\|}\right\|^{2} \leq 4\left(1-\|P(v)\|^{2}\right) .
$$

Proof. We have

$$
\left\|v-\frac{P(v)}{\|P(v)\|}\right\| \leq\|v-P(v)\|+\left\|P(v)-\frac{P(v)}{\|P(v)\|}\right\| \leq 2\|v-P(v)\|
$$

and

$$
\|v-P(v)\|^{2}=\langle v, v-P(v)\rangle=\langle v, v\rangle-\langle v, P(v)\rangle=1-\|P(v)\|^{2} .
$$

Proof of Proposition 4.1. The proof is by induction on $k$.

(1) We show that $\mathrm{A}$ is true for $k=1$.

(2) We show that if $\mathrm{A}$ is true for $k$, then $\mathrm{B}$ is true for $k$.

(3) We show that if $\mathrm{A}$ and $\mathrm{B}$ are true for $1, \ldots, k$, then $\mathrm{C}$ is true for $k$.

(4) We show that if $\mathrm{A}, \mathrm{B}, \mathrm{C}$ are true for $1, \ldots, k-1$, then $\mathrm{A}$ is true for $k$.

So because $\mathrm{A}$ is true for $k=1$, it follows that $\mathrm{B}$ and $\mathrm{C}$ are true for $k=1$, and this implies that $\mathrm{A}$ is true for $k=2$. Then the induction continues in the obvious way.

(1) Proof of A for $k=1$ : this follows directly from Proposition 4.2 with $A_{1}=2$.

(2) Proof of A true for k implies B for k: let $f^{\prime} \in E_{k}^{\prime},\left\|f^{\prime}\right\|=1$. If $\delta_{N_{k-1}+1} \leq$ $\frac{\Lambda_{k}}{2 A_{k}}$, the restriction of $P_{k}$ to $E_{k}$ is bijective and there exist $f \in E_{k},\|f\|=1$ with $f^{\prime}=\frac{P_{k}(f)}{\left\|P_{k}(f)\right\|}$. It follows from Lemma 4.1 that $\left\|f^{\prime}-f\right\|^{2} \leq 4\left\|\left(I-P_{k}\right)(f)\right\|^{2} \leq$ $4 \frac{A_{k} \delta_{N_{k-1}+1}}{\Lambda_{k}}$.

(3) Proof of A and B true for $1, \ldots, k$ implies $\mathrm{C}$ for $k$.

Let $f \in E_{k+l}, l \geq 1$ and $\|f\|=1$. Let $i \leq k$. For any $h^{\prime} \in E_{i}^{\prime},\left\|h^{\prime}\right\|=1$, because $P_{i}$ is surjective under the hypothesis $\delta_{N_{i}+1} \leq \frac{\Lambda_{i}}{2 A_{i}}$, there exist $h \in E_{i}$, $\|h\|=1$ with $h^{\prime}=\frac{P_{i}(h)}{\left\|P_{i}(h)\right\|}$. We have

$$
\left\langle P_{i}(f), h^{\prime}\right\rangle=\left\langle f, h^{\prime}\right\rangle=\left\langle f, h^{\prime}-h\right\rangle+\langle f, h\rangle .
$$


As in the case $k=1$, we have $\langle f, h\rangle=0$, so

$$
\left\langle P_{i}(f), h^{\prime}\right\rangle=\left\langle f, h^{\prime}-h\right\rangle \leq\|f\|\left\|h^{\prime}-h\right\|=\left\|h-\frac{P_{i}(h)}{\left\|P_{i}(h)\right\|}\right\| .
$$

By Lemma 4.1, we get $\left|\left\langle P_{i}(f), h^{\prime}\right\rangle\right|^{2} \leq 4\left(1-\left\|P_{i}(h)\right\|^{2}\right) \leq 4 A_{i} \frac{\delta_{N_{i-1}+1}}{\Lambda_{i}}$ by A. Using $\frac{\delta_{N_{i-1}+1}}{\Lambda_{i}} \leq \frac{\delta_{N_{k-1}+1}}{\Lambda_{k}}$, and because this is true for each $h^{\prime} \in E_{i}^{\prime},\left\|h^{\prime}\right\|=1$, we have

$$
\left\|P_{i}(f)\right\|^{2} \leq 4 A_{i} \frac{\delta_{N_{k-1}+1}}{\Lambda_{k}}
$$

and we deduce that

$$
\sum_{i=1}^{k}\left\|P_{i}(f)\right\|^{2} \leq 4\left(\sum_{i=1}^{k} A_{i}\right) \frac{\delta_{N_{k-1}+1}}{\Lambda_{k}} .
$$

(4) Proof of A, B, C true for $1, \ldots, k-1$ implies A for $k$.

Note that, because we have $A_{i} \geq 2$, the hypothesis $\delta_{N_{k}+1} \leq \frac{\Lambda_{k}}{2 A_{k}}$ implies $\delta_{N_{k}+1} \leq$ $\frac{\Lambda_{k}}{2}$. By Proposition 4.2, we have for $f \in E_{k},\|f\|=1$, and under the condition $\delta_{N_{k}+1} \leq \frac{\Lambda_{k}}{2}$,

$$
\left\|\left(I-P_{k}\right)(f)\right\|^{2} \leq \frac{2}{\Lambda_{k}}\left(\delta_{N_{k-1}+1}+\lambda_{N_{k}+1}^{\prime}\left\|P_{1}(f)+\cdots+P_{k-1}(f)\right\|^{2}\right) .
$$

By induction, $\left\|P_{1}(f)+\cdots+P_{k-1}(f)\right\|^{2} \leq 4\left(\sum_{i=1}^{k-1} A_{i}\right) \frac{\delta_{N_{k-2}+1}}{\Lambda_{k-1}}$, which is by definition $\leq 4\left(\sum_{i=1}^{k-1} A_{i}\right) \frac{\delta_{N_{k-1}+1}}{\Lambda_{k}}$.

So we choose $A_{k}=2+8 \frac{\lambda_{N_{k-1}+1} \sum_{i=1}^{k-1} A_{i}}{\Lambda_{k}}$.

\section{Proof of Lemma 2.1}

The proof of Lemma 2.1 is very close to the proof of Lemma 3.13 in [3]. Under the hypothesis

$$
\left|\left\langle\psi_{i}, \psi_{j}\right\rangle-\delta_{i, j}\right| \leq \rho, \quad q\left(\psi_{i}\right) \leq \lambda_{i}(1+\rho),
$$

for $1 \leq i, j \leq k$, we prove that

$$
q\left(F_{i}\right) \leq \lambda_{i}\left(1+\rho b_{k}\right) .
$$

Here the constants $b_{k}$ are defined by

$$
b_{k}=\left(1+8 a_{k}\right)\left(1+\left(1+\rho b_{k-1}\right)\left(1+8 a_{k}\right)\right)
$$


(where the $a_{k}$ are defined in the statement of the lemma) with $b_{1}=4$, and $\left\{F_{i}\right\}_{i=1}^{k}$ is the orthonormal basis naturally associated to the basis $\left\{\psi_{i}\right\}_{i=1}^{k}$. We have

where

$$
F_{i}=\frac{h_{i}}{\left\|h_{i}\right\|}
$$

$$
h_{i}=\psi_{i}-\sum_{j=1}^{i-1}\left\langle F_{j}, \psi_{i}\right\rangle F_{j}
$$

In particular, $b_{k}$ depends only on $k$ (and not on $\lambda_{k}$ ), but in a rather complicated form. We also denote by $p\left(\psi_{i}\right)$ the projection of $\psi_{i}$ given by $p\left(\psi_{i}\right)=\sum_{j=1}^{i-1}\left\langle F_{j}, \psi_{i}\right\rangle F_{j}$, so that we have the relation $\psi_{i}=h_{i}+p\left(\psi_{i}\right)$.

Proof. First part. The beginning of the proof is verbatim the same as the proof of Lemma 3.13 in [3].

We suppose the result is true for $s<k$ and show it for $s=k$. As a first step, note that

$$
q\left(F_{1}\right)=\frac{q\left(\psi_{1}\right)}{\left\|\psi_{1}\right\|^{2}} \leq \lambda_{1} \frac{1+\rho}{1-\rho}=\lambda_{1}\left(1+\rho \frac{2}{1-\rho}\right),
$$

so using $\rho<\frac{1}{2}$ we can take $b_{1}=4$. We also use without repeating the proof the following fact proved in the first part of Lemma 3.13 in [3]. For $i=1, \ldots, k-1$ and $s>i$, we have

$$
\left|\left\langle\psi_{s}, F_{i}\right\rangle\right| \leq \sqrt{2} a_{i} \rho
$$

We have for $s<k$

$$
q\left(p\left(\psi_{s}\right)\right)=\sum_{j=1}^{s-1} \sum_{l=1}^{s-1}\left\langle\psi_{s}, F_{j}\right\rangle\left\langle\psi_{s}, F_{l}\right\rangle q\left(F_{j}, F_{l}\right) .
$$

By the recurrence hypothesis we have

$$
\begin{aligned}
q\left(F_{j}, F_{l}\right) & \leq q^{1 / 2}\left(F_{j}\right) q^{1 / 2}\left(F_{l}\right) \\
& \leq\left(\lambda_{j}\left(1+\rho b_{j}\right)\right)^{1 / 2}\left(\lambda_{l}\left(1+\rho b_{l}\right)\right)^{1 / 2} \leq \lambda_{s-1}\left(1+\rho b_{s-1}\right),
\end{aligned}
$$

and, using the definition of the $a_{j}$ along with the Cauchy-Schwarz inequality,

$$
q\left(p\left(\psi_{s}\right)\right) \leq \lambda_{s-1}\left(1+\rho b_{s-1}\right) 2 \rho^{2} \sum_{j, l=1}^{s-1} a_{j} a_{l} \leq 2 \rho^{2} a_{s} \lambda_{s-1}\left(1+\rho b_{s-1}\right) .
$$

We will also use the fact that $\left\|h_{s}\right\|^{2} \geq 1-\rho a_{s}$. This implies (and this is the main change)

$$
\frac{1}{\left\|h_{s}\right\|^{2}} \leq 1+2 \rho a_{s}
$$


Second part. The following includes some new developments in comparison with Lemma 3.13 in [3].

We use that for $2 \leq s \leq k$

$$
q\left(F_{s}\right) \leq \frac{1}{\left\|h_{s}\right\|^{2}}\left(q\left(\psi_{s}\right)+q\left(p\left(\psi_{s}\right)\right)+2 \sqrt{q\left(\psi_{s}\right) q\left(p\left(\psi_{s}\right)\right)}\right.
$$

and get the following estimate for $q\left(F_{k}\right)$ :

$$
\begin{aligned}
q\left(F_{k}\right) \leq\left(1+2 \rho a_{k}\right)\left[\lambda_{k}\right. & (1+\rho)+\lambda_{k-1} 2 \rho^{2} a_{k}\left(1+\rho b_{k-1}\right) \\
& \left.+2 \lambda_{k}^{1 / 2} \lambda_{k-1}^{1 / 2}(1+\rho)^{1 / 2}\left(2 a_{k}\left(1+\rho b_{k-1}\right)\right)^{1 / 2} \rho\right] .
\end{aligned}
$$

Because $\lambda_{k-1} \leq \lambda_{k}$, we get

$$
\begin{aligned}
q\left(F_{k}\right) \leq \lambda_{k}\left(1+2 \rho a_{k}\right)[(1 & +\rho)+2 \rho^{2} a_{k}\left(1+\rho b_{k-1}\right) \\
& \left.+2(1+\rho)^{1 / 2}\left(2 a_{k}\left(1+\rho b_{k-1}\right)\right)^{1 / 2} \rho\right] .
\end{aligned}
$$

But

$$
\begin{aligned}
& \left(1+2 \rho a_{k}\right)\left[(1+\rho)+2 \rho^{2} a_{k}\left(1+\rho b_{k-1}\right)\right. \\
& \left.+2(1+\rho)^{1 / 2}\left(2 a_{k}\left(1+\rho b_{k-1}\right)\right)^{1 / 2} \rho\right] \\
& =1+\rho 2 a_{k}\left[(1+\rho)+2 \rho^{2} a_{k}\left(1+\rho b_{k-1}\right)\right. \\
& \left.\quad+2(1+\rho)^{1 / 2}\left(2 a_{k}\left(1+\rho b_{k-1}\right)\right)^{1 / 2} \rho\right] \\
& +\rho\left[1+2 \rho a_{k}\left(1+\rho b_{k-1}\right)+2(1+\rho)^{1 / 2}\left(2 a_{k}\left(1+\rho b_{k-1}\right)\right)^{1 / 2}\right] .
\end{aligned}
$$

So to obtain the conclusion, we have to show that

$$
\begin{gathered}
b_{k} \geq 2 a_{k}\left[(1+\rho)+2 \rho^{2} a_{k}\left(1+\rho b_{k-1}\right)+2(1+\rho)^{1 / 2}\left(2 a_{k}\left(1+\rho b_{k-1}\right)\right)^{1 / 2} \rho\right] \\
+\left[1+2 \rho a_{k}\left(1+\rho b_{k-1}\right)+2(1+\rho)^{1 / 2}\left(2 a_{k}\left(1+\rho b_{k-1}\right)\right)^{1 / 2}\right] .
\end{gathered}
$$

We simplify this expression using inequalities implied by the definition of $\rho$ and $a_{k}$ such as $\rho<1 / 2,2 \rho^{2} a_{k}<1,2 \rho a_{k} \leq 1$ along with $\sqrt{x} \leq x$ if $x \geq 1$. This gives

$$
\begin{aligned}
& {\left[(1+\rho)+2 \rho^{2} a_{k}\left(1+\rho b_{k-1}\right)+2(1+\rho)^{1 / 2}\left(2 a_{k}\left(1+\rho b_{k-1}\right)\right)^{1 / 2} \rho\right]} \\
& \quad \leq\left[(1+\rho)+2 \rho^{2} a_{k}\left(1+\rho b_{k-1}\right)+2(1+\rho)\left(2 a_{k}\left(1+\rho b_{k-1}\right)\right) \rho\right] \\
& \quad \leq\left[(1+\rho)+\left(1+\rho b_{k-1}\right)\left(2 \rho^{2} a_{k}+4(1+\rho) a_{k}\right)\right] \\
& \quad \leq 2+\left(1+\rho b_{k-1}\right)\left(1+8 a_{k}\right)
\end{aligned}
$$

and

$$
\begin{aligned}
{[1} & \left.+2 \rho a_{k}\left(1+\rho b_{k-1}\right)+2(1+\rho)^{1 / 2}\left(2 a_{k}\left(1+\rho b_{k-1}\right)\right)^{1 / 2}\right] \\
& \leq\left[1+2 \rho a_{k}\left(1+\rho b_{k-1}\right)+2(1+\rho)\left(2 a_{k}\left(1+\rho b_{k-1}\right)\right)\right] \\
& =1+\left(1+\rho b_{k-1}\right)\left[2 \rho a_{k}+4(1+\rho) a_{k}\right] \\
& \leq 1+\left(1+8 a_{k}\right)\left(1+\rho b_{k-1}\right) .
\end{aligned}
$$


We can then finish with

$$
\begin{aligned}
2 a_{k} & {\left[2+\left(1+\rho b_{k-1}\right)\left(1+8 a_{k}\right)\right]+\left[1+\left(1+8 a_{k}\right)\left(1+\rho b_{k-1}\right)\right] } \\
& =\left(1+\rho b_{k-1}\right)\left(1+8 a_{k}\right)\left(1+2 a_{k}\right)+1+4 a_{k} \\
& \leq\left(1+\rho b_{k-1}\right)\left(1+8 a_{k}\right)\left(1+8 a_{k}\right)+1+8 a_{k} \\
& =\left(1+8 a_{k}\right)\left(1+\left(1+\rho b_{k-1}\right)\left(1+8 a_{k}\right)\right) \\
& \leq b_{k}
\end{aligned}
$$

using that the assumption (1.2) of Theorem 1.1 implies $\rho \leq 1$.

\section{References}

[1] A. Ancona, On strong barriers and an inequality of Hardy for domains in $\mathbb{R}^{n}$. J. London Math. Soc. (2) 2 (1986), 274-290. MR 0856511 Zbl 0629.31002

[2] M. van den Berg, On the asymptotics of the heat equation and bounds on traces associated with the Dirichlet Laplacian. J. Funct. Anal. 71 (1987), 279-293. MR 0880981 Zbl 0632.35016

[3] J. Bertrand and B. Colbois, Capacité et inégalité de Faber-Krahn dans $\mathbb{R}^{n}$. J. Funct. Anal. 232 (2006) 1-28. MR 2200165 Zbl 1100.31005

[4] V. I. Burenkov, P. D. Lamberti, and M. Lanza de Cristoforis, Spectral stability of nonnegative selfadjoint operators. Sovrem. Mat. Fundam. Napravl. 15 (2006), 76-111. English transl.: J. Math Sci (N.Y.) 149 (2008), 1417-1452. MR 2336430

[5] S.Y. Cheng, Eigenvalue comparison theorems and its geometric applications. Math. Z. 143 (1972), 289-297. MR 0378001 Zbl 0329.53035

[6] D. Daners, Dirichlet problems on varying domains. J. Differential Equations 188 (2003), 591-624. MR 1955096 Zbl 1090.35069

[7] E. B. Davies, Heat kernels and spectral theory. Cambridge Tracts in Mathematics 92. Cambridge University Press, Cambridge, 1989. MR 0990239 Zbl 0699.35006

[8] E. B. Davies, Eigenvalue stability bounds via weighted Sobolev spaces. Math. Z. 214 (1993), 357-371. MR 1245198 Zbl 0799.58082

[9] E. B. Davies, Sharp boundary estimates for elliptic operators. Math. Proc. Cambridge Philos. Soc. 129 (2000) 165-178. MR 1757786 Zbl 0963.35146

[10] A. Henrot, Continuity with respect to the domain for the Laplacian: A survey. Control Cybernet. 23 (1994), 427-443. Special issue: J. Sokołowski (ed.), Shape design and optimization. MR $1303362 \mathrm{Zbl} 0822.35029$

[11] A. Henrot, Extremum problems for eigenvalues of elliptic operators. Frontiers in Mathematics. Birkhäuser Verlag, Basel, 2006. MR 2251558 Zbl 1109.35081

[12] M. Iversen, Variational problems: perturbations and optimal sets. Ph.D. thesis. University of Bristol, Bristol, 2013. 
[13] A. Lemenant and E. Milakis, Quantitative stability for the first Dirichlet eigenvalue in Reifenberg flat domains in $\mathbb{R}^{N}$. J. Math. Anal. Appl. 364 (2010), 522-533. MR 2576203 Zbl 1189.35203

[14] M. M. H. Pang, Approximation of ground state eigenvalues and eigenfunctions of Dirichlet Laplacians. Bull. London Math. Soc. 29 (1997), 720-730. MR 1468060 Zbl 0969.35102

Received June 22, 2012; revised December 11, 2012

Bruno Colbois, Institut de Mathématiques de Neuchâtel, rue Emile-Argand 11, 2009 Neuchâtel, Suisse

E-mail: bruno.colbois@unine.ch

Alexandre Girouard, Département de mathématiques et de statistique, Université Laval, Pavillon Alexandre-Vachon, 1045, av. de la Médecine, Québec (Qc) G1V 0A6, Canada

E-mail: alexandre.girouard@mat.ulaval.ca

Mette Iversen, School of Mathematics University Walk, Clifton, Bristol BS8 1TW, U.K.

E-mail: mette.iversen@cantab.net 